Brown Proc. natn. Acad. Sci. U.S.A. 75,$256 ; 1978$ ) possibly by means of a nuclease which degrades viral mRNA (Baglioni et al. Nature 273, 684; 1978). The last possibility provides a mechanism for interfering with virus replication in a manner related to that of interferons and their inducers but without actual production of interferon. The anti-viral effects of single-stranded polynucleotides which do not induce interferon may occur by this means or by mimicking strategic nucleotide sequences in viral RNA (Stebbing et al. Ann. N.Y. Acad. Sci. 284, 682; 1977). It is noteworthy that early studies on interferon induction by polynucleotides involved largely singlestranded RNAs (Isaacs et al. Lancet ii 113; 1963; Rotem et al. Nature 197, $564 ; 1963)$ and that two separate mechanisms may have been involved in the overall anti-viral effect obtained. Induction of interferon was certainly a factor but independent more direct antiviral effects may also have been involved.

\section{Flattening of Uranus and Neptune}

from David $W$. Hughes

Planetary fiattening or oblateness, $\varepsilon$, is defined as the difference between the equatorial and polar radii divided by the equatorial radius. It is not an easy quantity to determine especially in the case of the outer planets. The flattening can be obtained geometrically by using for example a double image micrometer in the focal plane of a large telescope or by the analysis of the isophotes of the image of the planet obtained from spacecraft photographs. It can be measured dynamically by observing the orbital perturbations of the inner satellites of the planet induced by its unsymmetrical mass distribution, calculating $J_{2}$, the quadruple moment of the gravitational field and using the simple relationship between this quantity and the flattening $\varepsilon$. Unfortunately if we take the fairly typical case of Uranus, the inner satellites Miranda, Ariel and Umbriel form a system with near commensurability of mean motions, a state of affairs that results in important mutual perturbations.

A. H. Cook of the Cavendish Labora. tory, University of Cambridge reviews recent observations of $\varepsilon$ in the light of the new determinations of the spin periods of the planets and shows that some of the values obtained for $\varepsilon$ are implausible to say the least. His conclusions are presented in a recent edition of the Monthly Notices of the Royal Astronomical Society (187, 39P;

David $W$. Hughes is a Lecturer in the Department of Physics at the University of Sheffield.
1979).

For a planet in hydrostatic equilibrium the product $C / M a^{2}$, where $C$ is the moment of inertia about the polar axis, $M$ is the mass and $a$ is the radius, must be between specific limits. This quantity is 0.4 if the planet is of uniform density and drops to 0 for an extreme central condensation.

Unfortunately the present day values obtained for the dynamical properties of Uranus and Neptune are confusingly diverse. For Uranus, recent measurements of the spin period give two inconsistent values, one about $24 \mathrm{~h}$ and the other about $14.5 \mathrm{~h}$. There are two values for Neptune as well, one about $22 \mathrm{~h}$ and the other about $18.5 \mathrm{~h}$. Inconsistent values have been found for one or both planets for $\varepsilon$ and $J_{2}$.

Cook then considers all possible combinations of the observed parameters to see which if any can be rejected due to the physical unreality of the value of $C / M a$ so produced. The central pressures of Uranus and Neptune are comparable with those of the Earth, however the mean molecular weights of the material in these planets are less and the material is more compressible. Cook concludes that these planets have $\mathrm{C} / \mathrm{Ma}$ values which are definitely greater than 0.25 and probably in excess of 0.3 . On this basis both the values obtained for the geometrical flattening of Uranus, 0.028 and 0.01 , are inadmissable. It is not possible to decide between the three $J_{2}$ values of 0.00343 , 0.005 and 0.012 . For Neptune the $\varepsilon$ and $J_{2}$ values both support the lower spin period of $18.5 \mathrm{~h}$.

To resolve this sorry state of affairs the astronomical community must be stimulated into taking new observations. Unfortunately, as is so often the case, the best place to take these observations is outside the distorting a.mosphere of planet Earth.

\section{More spots before the eyes}

\section{from E. G. Richards}

TREATMENT of Escherichia coli DNA with $E c o \mathrm{R} 1$ restriction enzyme results in an estimated 500-600 fragments of DNA differing first in their molecular size and second in their base composition and sequence. If such a digest is examined by electrophoresis in agarose, a technique which separates DNA according to molecular size, a relatively featureless pattern is obtained, for the resolving power of the method is insufficient to resolve such a large number of components. How useful it would be, however, if this were possible.

Fischer and Lerman (Cell 16, 191;
1979) have recently described a twodimensional electrophoretic method which approaches this ideal. They start with the observation that a mixture of urea and formamide-both non-ionic substances-can denature DNA. That is to say, if the concentration of these denaturants is increased, a point is reached at which the DNA wholly or completely 'melts'. This critical concentration depends on the temperature, occurring at a lower concentration the higher the temperature, and also on the base composition and sequence, albeit in a complicated way.

In their first experiment Fischer and Lerman applied a sample of an EcoR1 digest of $\lambda$ phage DNA, containing six fragments, along the top of a slab polyacrylamide gel and subjected it to electrophoresis downwards. They had, however, arranged that the gel contained a gradient of concentration of urea and formamide in a direction perpendicular to the direction of migration. After electrophoresis they observed that in the part of the gel where denaturant concentration was low the six fragments were resolved according to molecular size and moved with a velocity almost independent of denaturant concentration. On the other hand, on the high-concentration side of the gel the components moved with a velocity reduced by a factor of 20 or 30 and remained near the top of the gel. At intermediate parts of the gel where the denaturant concentration took intermediate values, the components moved with intermediate velocities. The result was that the zone of DNA corresponding to each fragment was distorted into a sigmoidal curve resembling a 'melting curve'. Corresponding to each fragment there was a critical denaturant concentration at which the mobility fell to a low value and at which partial or complete denaturation took place. This critical concentration varied from one fragment to another and no doubt depended on the composition and sequence of the fragment. If the temperature at which the gel was maintained was reduced (from $60{ }^{\circ} \mathrm{C}$ to $45^{\circ} \mathrm{C}$ ) this critical concentration increased as expected. It may be noted that this simple description was slightly complicated for one or two fragments by the presence of more than one step in the 'melting curve', a phenomenon probably associated with the denaturation of separate domains of the DNA at different denaturant concentrations.

These observations are certainly entertaining but not of great apparent importance. This importance, however, is provided in the next and crucial stage of Fischer and Lerman's work when they rotated the denaturant gradient through $90^{\circ}$ so that the concentration of urea and formamide increased in the direction of migration. They then ob- 